\title{
In Vitro Demonstration of Intestinal Absorption Mechanisms of Different Sugars Using 3D Organotypic Tissues in a Fluidic Device
}

\author{
Alessandra Marrella 1 , Paolo Buratti ${ }^{1}$, Jan Markus ${ }^{2}$, Giuseppe Firpo ${ }^{3}$, Mario Pesenti $^{4}$, Timothy Landry 5 , \\ Seyoum Ayehunie ${ }^{5}$, Silvia Scaglione ${ }^{1,6}$, Helena Kandarova ${ }^{7}$ and Maurizio Aiello 6 \\ ${ }^{1}$ CNR-IEIIT Institute, National Research Council of Italy, Genoa, Italy; ${ }^{2}$ MatTek In Vitro Life Science Laboratories, Bratislava, Slovakia; ${ }^{3}$ Department \\ of Physics, University of Genoa, Genoa, Italy; ${ }^{4}$ Gastroenterology Unit, Department of Internal Medicine, Ospedale Policlinico IRCCS San Martino \\ University of Genoa, Genoa, Italy; ${ }^{5}$ MatTek Corporation, Ashland, MA, USA; ${ }^{6}$ React4life S.r.1., Genoa, Italy; ${ }^{7}$ Institute of Experimental Pharmacology \\ and Toxicology, Centre of Experimental Medicine, Slovak Academy of Sciences, Bratislava, Slovakia
}

\begin{abstract}
Intestinal permeability is crucial in regulating the bioavailability and, consequently, the biological effects of drugs and compounds. However, systematic and quantitative studies of the absorption of molecules are quite limited due to a lack of reliable experimental models able to mimic human in vivo responses. In this work, we present an in vitro perfused model of the small intestinal barrier using a 3D reconstructed intestinal epithelium integrated into a fluid-dynamic bioreactor $\left(M^{\prime} \mathrm{VO}^{\circledR}\right)$ resembling the physiological stimuli of the intestinal environment. This platform was investigated in both healthy and induced pathological conditions by monitoring the absorption of two non-metabolized sugars, lactulose and mannitol, frequently used as indicators of intestinal barrier dysfunctions. In healthy conditions, an in vivo-like plateau of the percentage of absorbed sugars was reached, where mannitol absorption was much greater than lactulose absorption. Moreover, a model of pathologically altered intestinal permeability was generated by depleting extracellular $\mathrm{Ca}^{2+}$, using a calcium-specific chelator. After calcium depletion, the pattern of sugar passage observed under pathological conditions was reversed only in dynamic conditions in the MIVO ${ }^{\circledR}$ chamber, due to the dynamic fluid flow beneath the membrane, but not in static conditions. Therefore, the combination of the $\mathrm{MIVO}^{\circledR}$ with the Epilntestinal ${ }^{\mathrm{TM}}$ platform can represent a reliable in vitro model to study the passage of molecules across the healthy or pathological small intestinal barrier by discriminating the two main mechanisms of intestinal absorption.
\end{abstract}

\section{Introduction}

The intestinal epithelium is the main access point of nutrients and orally administered drugs to the systemic circulation (Ganapathy, 2012). Molecules can pass through the intestinal barrier by two main parallel routes: the transcellular pathway, in which drugs are transported through the cell membrane, and the paracellular pathway, in which drugs pass through the intercellular spaces (Groschwitz and Hogan, 2009). Studies on the intestinal absorption of molecules that consider the relative importance of these two alternative routes of entry are often limited due to a lack of validated or widely accepted experimental models (Stewart et al., 1995).

The use of animal models to evaluate gastrointestinal (GI) toxicity often fails to uncover the drug toxicities seen after human use. Concerns related to the currently available in vivo models for drug permeation studies include species differences, ethical issues, and high cost of animal experiments (Holmes et al., 2010). Although animal-based tests reproduce the dynamic stimuli of the intestinal environment mediated by the systemic blood circulation, it is impossible to selectively distinguish and decouple all the variables involved in the passage of molecules through the intestinal barrier in human intestinal tissues (Cao et al., 2006). For these reasons, there is a growing interest to develop advanced, human-relevant in vitro models, where key parameters such as intestinal permeability and tissue barrier properties can be easily controlled and monitored at low cost and reduced time.

In vitro models of human intestinal epithelium are mostly based on the static culture of intestinal epithelial cell monolayers derived from colon adenocarcinoma (e.g., Caco-2), which do
Received August 31, 2019; Accepted December 18, 2019; Epub December 30, 2019; () The Authors, 2019

ALTEX 37(2), 255-264. doi:10.14573/altex.1908311

Correspondence: Silvia Scaglione, $\mathrm{PhD}$

CNR - IEIIT Institute, National Research Council of Italy

Via De Marini 6, 16149 Genova, Italy

(silvia.scaglione@ieiit.cnr.it)
This is an Open Access article distributed under the terms of the Creative Commons Attribution 4.0 International license (http://creativecommons.org/licenses/by/4.0/) which permits unrestricted use, distribution and reproduction in any medium, provided the original work is appropriately cited. 
not reproduce the production of mucus or differentiate into villi-like structures to recapitulate the physiology of the human intestine. Also, Caco-2 cultures form a non-physiological barrier due to paracellular junctions that are much tighter and less permeable than those of native human intestinal epithelium (Ayehunie et al., 2018). The average tight junction pore radius of $\mathrm{Ca}-$ co- 2 cells is estimated to be $3.7 \AA$, compared to $8-13 \AA$ typical of native human tissue (Tavelin et al., 2003). Moreover, transepithelial electrical resistance (TEER) measurements for Caco-2 monolayers/tissues range from 250 to $2400 \Omega * \mathrm{~cm}^{2}$ compared to TEER values of $12-120 \Omega * \mathrm{~cm}^{2}$ for human small intestinal tissue (Gupta et al., 2013; Tavelin et al., 2003). Caco-2 cells also have other limitations such as: (i) weak or no expression of important intestinal metabolic enzymes such as cytochrome P450 (CYP3A) (Le Ferrec et al., 2001), (ii) lack of the crypt-villus axis, which is important for fluid and ion transport, and (iii) variability in cell line and clone selection, which can lead to 20 -fold differences in TEER from laboratory to laboratory (Huang and Adams, 2003). Despite the recent efforts in establishing alternatives to animal tests for intestinal absorption, there is still a lack of reliable models with physiological relevance (Marrella et al., 2018).

Recently, 3D human intestinal cultures were developed to overcome these limitations. Such intestinal tissue models utilize primary human intestinal cells to produce a highly differentiated and polarized epithelial structure including the different cellular phenotypes such as enterocytes, MUC2+ (goblet) cells, Paneth cells, enteroendocrine cells, and tuft cells. This 3D EpiIntestinal ${ }^{\mathrm{TM}}$ tissue model can be cultured over 6 weeks, which makes it ideal to run long term and repeat/chronic dosing experiments. The model is highly reproducible with lower lab-to-lab variation than for Caco-2 (Markus, et al., 2017). EpiIntestinal ${ }^{\mathrm{TM}}$ tissues have morphological and ultrastructural features such as villi-like structures and brush border formation that resembles normal human intestinal epithelium (Ayehunie et al., 2018). However, these $3 \mathrm{D}$ reconstructed tissues are usually static and therefore lack physiological fluidic stimuli of the living microenvironment (Benam et al., 2015).

Therefore, in this study, the EpiIntestinal ${ }^{\mathrm{TM}}$ tissue was integrated into a fluidic bioreactor commercialized as $\mathrm{MIVO}^{\circledR}$ by React4life S.r.l. Genova, Italy. This novel in vitro platform combines the advantages of 3D human small intestinal tissue and flow conditions that mimic the physiological microenvironment of the gut. The platform allows the quantitative biochemical analysis of permeability/metabolism of molecules (e.g., lactulose and mannitol) over time as well as the morphological characterization of the 3D tissue cultured in a sterile environment under either static or dynamic conditions.

Two sugar molecules commonly used in the clinic to identify intestinal barrier disruption were used to characterize the model: Mannitol is a small molecule (molecular diameter of 6.5 $\AA$; MW: $182.172 \mathrm{~g} / \mathrm{mol}$ ) that is mainly transported through the pores on the villi surfaces (transcellularly), although it may also pass across the intestinal epithelium via paracellular transport, especially after tight junction disruption, due to its small size. In contrast, lactulose is a disaccharide (molecular diameter: $9.5 \AA$;
MW: $342.296 \mathrm{~g} / \mathrm{mol}$ ) that moves through larger spaces between the cells (Bjarnason et al., 1995; Dastych et al., 2008; Sequeira et al., 2014) by passive diffusion.

Perturbation or disruption of the intestinal barrier by compounds or biomolecules can compromise intestinal barrier function and allow microbial entry, which can lead to pathologic conditions including celiac disease (Collares-Buzato et al., 1994), Crohn's disease (Söderholm et al., 1999) and inflammatory bowel disease (Halme et al., 2000). To investigate whether the model reflects changes in intestinal absorption under pathological conditions and also barrier recovery, a model of altered intestinal permeability was generated via calcium chelation with EGTA (Collares-Buzato et al., 1994). The spontaneous ability of the intestinal epithelium to recover from this challenge was assessed by quantifying the change of lactulose and mannitol passage through the intestinal barrier.

Reconstructed small intestinal tissues cultured under flow conditions may be more predictive of in vivo molecule absorption than current in vitro or animal models. Hence, our approach contributes to the development of a reliable alternative to animal use, in accordance with the 3R (Reduce, Refine, Replace) principles described by Russell and Burch (Russell et al., 1959).

\section{Materials and methods}

\section{Cell source}

Primary human cells of the small intestine were obtained as previously described (Ayehunie et al., 2018). Briefly, cells were obtained from commercial sources or isolated from tissues obtained from the International Institute for the Advancement of Medicine (IIAM; Scranton, PA) through the Organ Procurement Organization (OPO). All tissue specimens were collected from braindead donors with informed consent from the next of kin. Samples were protected from ischemic injury by flushing with icecold University of Wisconsin storage solution immediately after vascular clamping and resection. Epithelial cells and fibroblasts were isolated from ileum, jejunum, or duodenum. Cells were expanded and cryopreserved for future use.

\section{Reconstructed in vitro $3 D$ tissue model of small

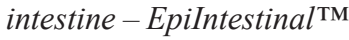

The human small intestine cells were obtained and used to produce a reconstructed tissue model as described before (Ayehunie et al., 2018). Briefly, cryopreserved intestinal epithelial cells were thawed and seeded onto collagen-coated cell culture inserts (MatTek Corporation, $0.6 \mathrm{~cm}^{2}$ ) in a proprietary medium formulation (SMI-100-MM, IVLSL, Bratislava, Slovakia). Cells were cultivated in submerged conditions for $24 \mathrm{~h}$, followed by cultivation for 13 days at the air-liquid interface (ALI) at $37^{\circ} \mathrm{C}$, $5 \% \mathrm{CO}_{2}$ and $98 \%$ relative humidity. During the ALI culture period, these tissues (designated SMI-100 or EpiIntestinal ${ }^{\mathrm{TM}}$ ) were fed basolaterally through the membrane of the cell culture inserts of the 24-well plate. To keep the apical layer moist/hydrated, $100 \mu \mathrm{L}$ of culture medium (MatTek Corporation) was applied topically every other day. To complete cellular differenti- 


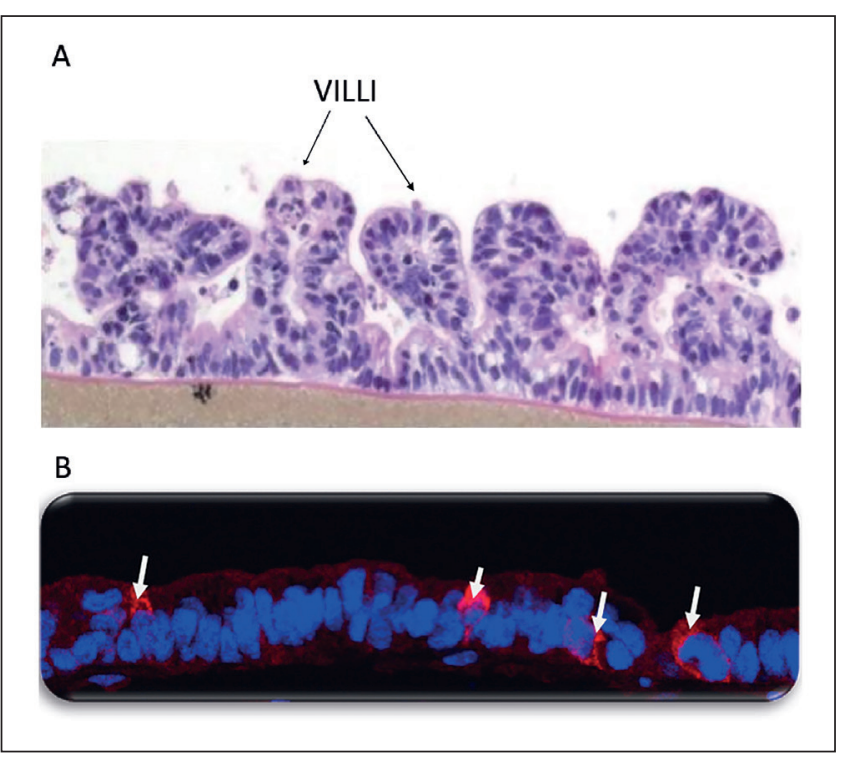

Fig.1: Typical histology of the Epilntestinal ${ }^{\mathrm{TM}}$ (SMI-100) model (H\&E staining) (A)

Immunohistochemistry showing MUC2+ mucus producing goblet cells (red) in intestinal tissues (white arrow). MUC-2 protein is a marker of mucous-producing goblet cells; DAPI positive nuclear staining is shown in blue $(B)$.

ation and stratification, cells were cultured for a total of 14 days prior to shipment and use to run the experiments within the bioreactor. Figure 1 shows the typical histology of the EpiIntestinal $^{\text {TM }}$ (SMI-100) model.

\section{MIVO fluidic chamber}

In vitro diffusion studies were performed using a compartmental fluidic device (commercialized as $\mathrm{MIVO}^{\circledR}$ by React4life S.r.1., IT). The diffusion system designed and implemented in this study is shown schematically in Figure 2.

MIVO $^{\circledR}$ is composed of a chamber made of transparent USPVI biopolymer and connected to a closed-loop fluidic circuit. The bioreactor fits commercially available 24-well trans-well inserts. Briefly, after $24 \mathrm{~h}$ of culture at ALI culture conditions to allow physiological (luminal) exposure conditions, 24-well EpiIntestinal ${ }^{\mathrm{TM}}$ inserts were placed into and cultured within the bioreactor, forming two fluidically independent chambers: the donor chamber, which was filled with $0.2 \mathrm{~mL}$ sugar solution (either lactulose or mannitol) at different concentrations, and the receiver chamber, connected to the fluidic circuit and filled with $2.5 \mathrm{~mL}$ phosphate buffered saline (PBS). The fluidic circuit was connected by paraben-free transparent tubes to a peristaltic pump (Watson Marlow), and PBS was pumped through the lower chamber at a rate of $2.3 \mathrm{~mL} / \mathrm{min}$, simulating the capillary flow rate $(0.1 \mathrm{~cm} / \mathrm{s})$. The PBS therefore flows in the basolateral chamber, beneath the membrane, in a closed-loop circuit, driven by the peristaltic pump (Fig. S1, S2 ${ }^{1}$ ).

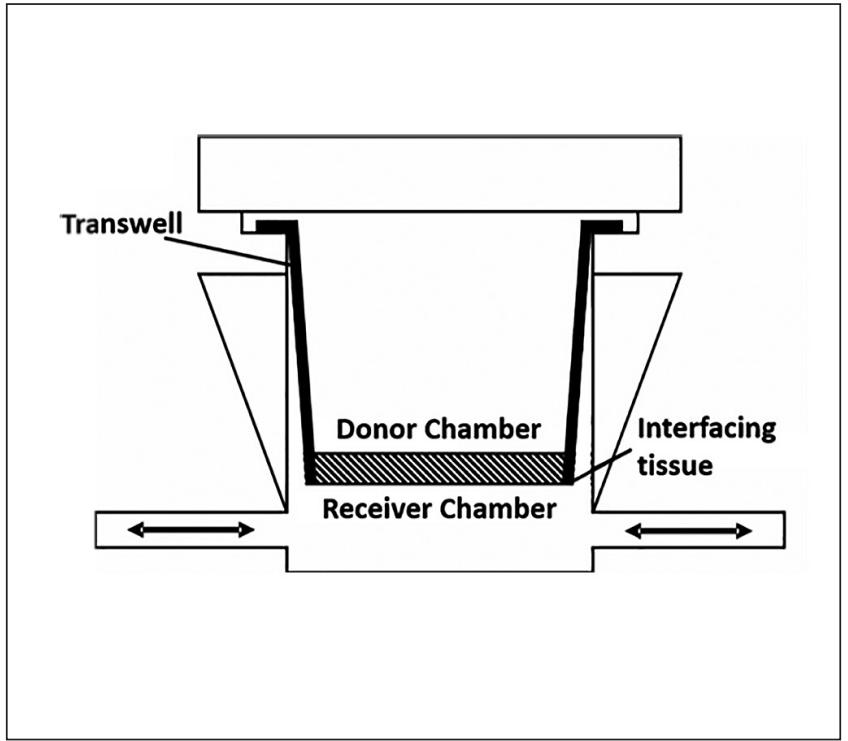

Fig. 2: Schematic representation of fluidic system and Epilntestinal ${ }^{\mathrm{TM}}$ tissue used as interfacing tissue within the $\mathrm{MIVO}^{\circledR}$ fluidic chamber for absorption tests

Transepithelial electrical resistance (TEER) measurement To obtain baseline data on tissue barrier, TEER was measured prior to exposure of tissues to lactulose or mannitol. TEER measurement was performed following the standard protocol provided by MatTek (Bratislava, Slovakia). Briefly, tissues were equilibrated with SMI-100-MM medium (MatTek) overnight prior to TEER measurement. Then tissues were transferred to TEER buffer $(100 \mathrm{mM} \mathrm{KCl})$ and TEER measurement was performed using an EVOM2 (World Precision Instruments, Sarasota, FL, USA) apparatus. To minimize any effect of TEER buffer, tissues were transferred back to medium and left to recover at $37^{\circ} \mathrm{C}$ for $25 \mathrm{~min}$.

\section{Permeability of lactulose and mannitol}

Intestinal barrier integrity was characterized by monitoring the absorption of the two non-metabolized sugars, lactulose and mannitol. Mannitol permeates through hydrophilic pores of the cell membrane (transcellular route) and lactulose is absorbed through the tight junctions (paracellular route).

$0.2 \mathrm{~mL}$ lactulose (Roche, Basel, Switzerland) was topically applied at 55.5 or $44.4 \mathrm{mg} / \mathrm{mL}$ for $30,60,90,120$, or $150 \mathrm{~min}$. At the end of each exposure time, the circulating solution in the receiver chamber was collected and the quantity of lactulose permeated through the epithelium was assessed spectrophotometrically by using a UV-3100PC UV/VIS scanning spectrophotometer (VWR International, Radnor, Pennsylvania, USA). Absorbance readings at $276 \mathrm{~nm}$ were used for quantification of the

\footnotetext{
1 doi:10.14573/altex.1908311s
} 


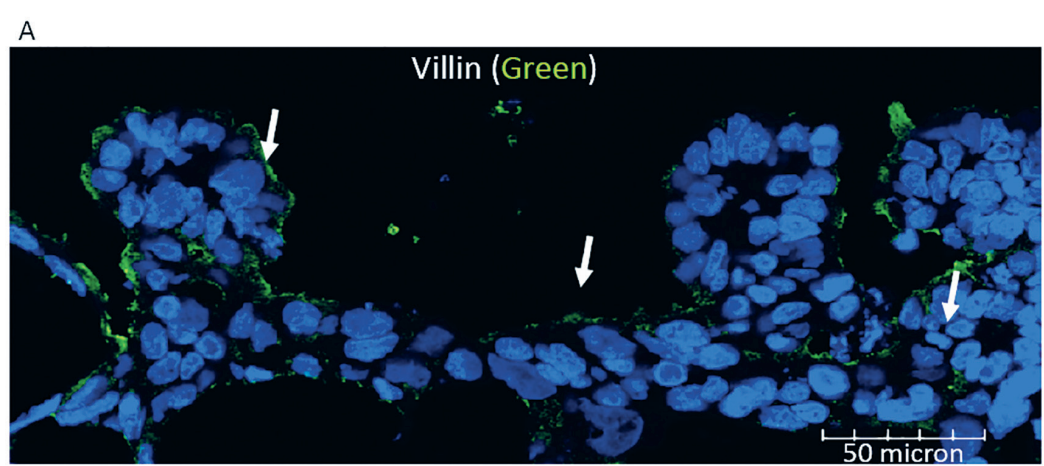

Fig. 3: Comparison of villin expression at the apical surfaces of intestinal tissues cultured under static (A) or dynamic (B) conditions in the MIVO ${ }^{\circledR}$ device Immunohistochemistry showing villin (green) expressing cells at the brush border (white arrows); DAPI positive nuclear staining is shown in blue.

B

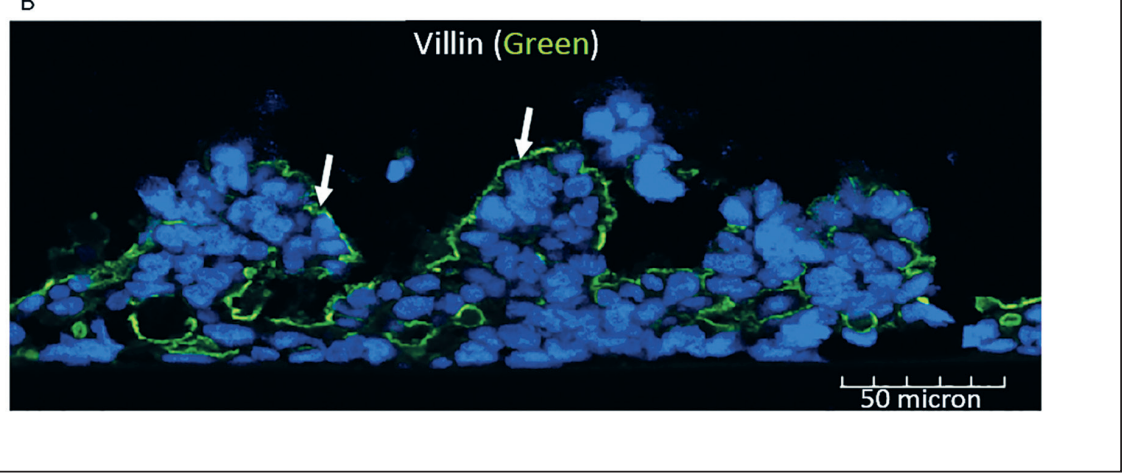

amount of lactulose passed through the intestinal tissues (via interpolation with a calibration curve).

$0.2 \mathrm{~mL}$ mannitol solution (Cat\# 282631, Dufour, Italy) was applied at 105 or $210 \mathrm{mg} / \mathrm{mL}$ as described previously (Dastych et al., 2008) for $30,60,90,120,150,180,210$, or $240 \mathrm{~min}$. At the end of each exposure time, the circulating solution in the receiver chamber was collected and the quantity of mannitol permeated through the intestine tissue was measured by using the K-MANOL assay kit from Megazyme (Bray, Co, Wiklow, Ireland) according to the manufacturer's instructions.

Lactulose and mannitol permeability were expressed in terms of permeation percentage relative to the applied quantity and by calculation of the lactulose/mannitol ratios (LMR).

\section{Model of the altered intestinal permeability via calcium \\ depletion and its recovery}

To alter the barrier properties of the EpiIntestinal ${ }^{\mathrm{TM}}$ model, extracellular $\mathrm{Ca}^{2+}$ was depleted by adding the calcium-specific chelator EGTA (ethylene glycol-bis( $\beta$-aminoethyl ether)$\mathrm{N}, \mathrm{N}, \mathrm{N}^{\prime}, \mathrm{N}^{\prime}$-tetraacetic acid), also known as egtazic acid), which is known to progressively disassemble tight junctions, the cellto-cell adhesions essential to maintain epithelial barrier integrity (Rothen-Rutishauser et al., 2002; Tsukita et al., 2001). Briefly, SMI-100-MM maintenance medium supplied with the tissues was supplemented with $25 \mathrm{mM}$ EGTA (Sigma Aldrich, Saint Louis, MO, USA) and added both to the apical and basolateral sides of EpiIntestinal ${ }^{\mathrm{TM}}$ tissues. After 45 min of incubation at Standard Culture Conditions (SCC), i.e., in a sterile humidified incubator at $37 \pm 1^{\circ} \mathrm{C}, 5 \pm 1 \% \mathrm{CO}_{2}, 95 \pm 5 \% \mathrm{RH}$, a
permeability test for lactulose and mannitol was carried out as described above.

To assess the ability of the tissues to repair the damage induced by calcium depletion, the EpiIntestinal ${ }^{\mathrm{TM}}$ inserts were then incubated for $24 \mathrm{~h}$ in SMI-100-MM maintenance medium at SCC. The tissues then again underwent permeability tests for lactulose and mannitol under static and dynamic conditions.

\section{Immunohistochemistry}

To examine structural features, EpiIntestinal ${ }^{\mathrm{TM}}$ tissues were fixed in $4 \%$ paraformaldehyde solution at $4{ }^{\circ} \mathrm{C}$ overnight, routinely processed, embedded in paraffin blocks, sectioned, and stained with hematoxylin and eosin. Immunohistochemical staining was performed on $5 \mu \mathrm{m}$ paraffin sections mounted on glass slides, deparaffinized, and processed for antigen retrieval by heating in a citrate buffer $\left(\mathrm{pH} \mathrm{7.4)}\right.$ for $45 \mathrm{~min}$ at $95-99^{\circ} \mathrm{C}$. After $45 \mathrm{~min}$, the specimens were removed from the heated water bath and left in the citrate buffer for an additional $20 \mathrm{~min}$ at RT. Rabbit polyclonal anti-villin ( $2 \mu \mathrm{g} / \mathrm{mL}$, cat\# ab97512, Abcam, Cambridge, MA) or anti-MUC2 antibody $(2 \mu \mathrm{g} / \mathrm{mL}$, cat\# ab76774, Abcam, Cambridge, MA) was applied for $60 \mathrm{~min}$ at RT and then visualized using secondary antibody $(5 \mu \mathrm{g} / \mathrm{mL}$, cat\#A-11008, Goat anti-Rabbit IgG $(\mathrm{H}+\mathrm{L})$ Cross-adsorbed AlexFluor 488 ThermoFisher, Waltham, MA)). DAPI (cat\#D1306, Life Technologies, Carlsbad, CA) was used for nuclear staining. Images were captured using a FV1000 confocal microscope (Olympus America Inc., Center Valley, PA). 


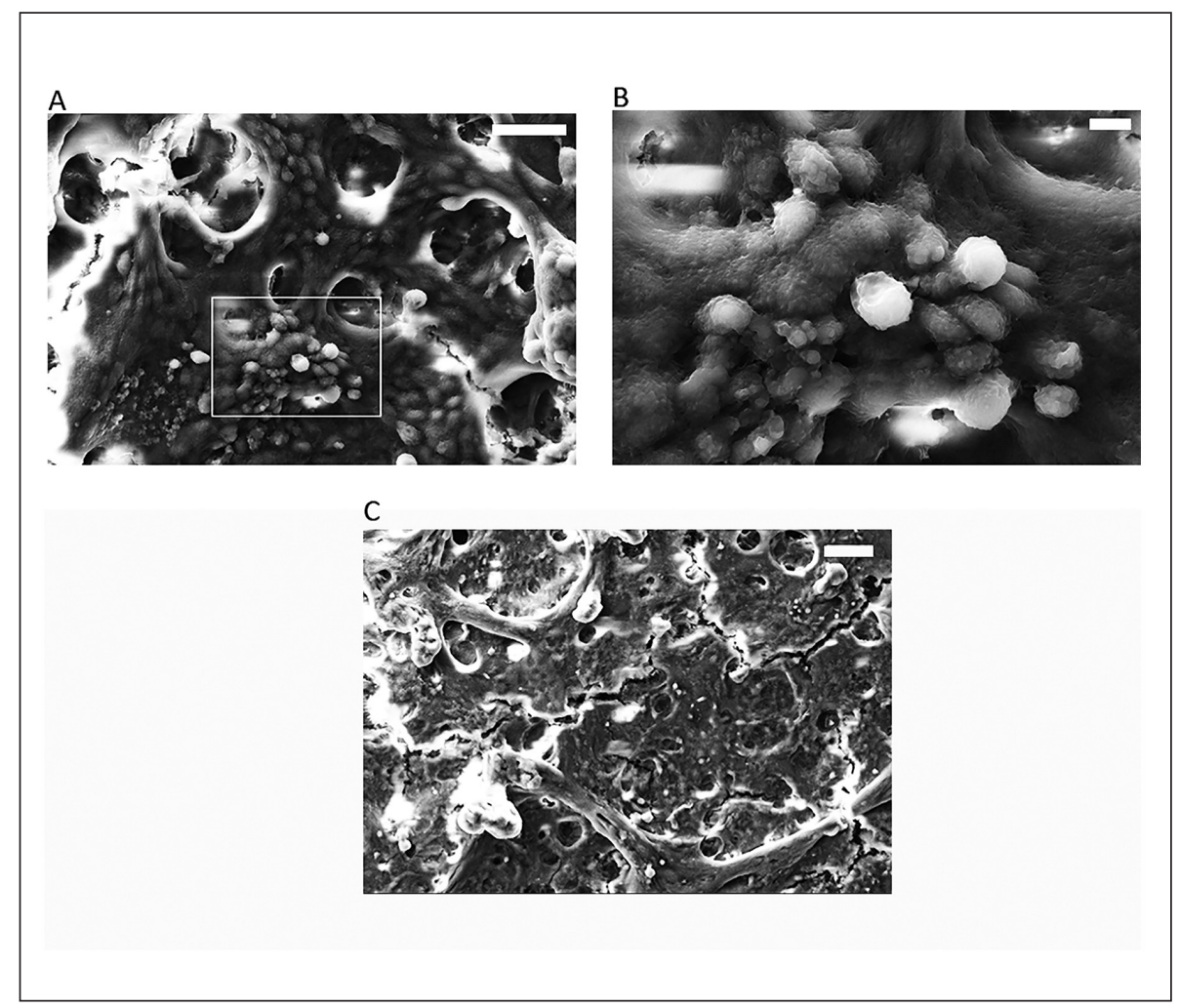

Fig. 4: FE-SEM analysis of intestinal tissue cultured within the $\mathrm{MIVO}^{\circledR}$ chamber under dynamic (Panels A, B) and static (C) conditions Bar is $30 \mu \mathrm{m}$ (A), $5 \mu \mathrm{m}$ (B), $40 \mu \mathrm{m}$ (C).

\section{FE-SEM analysis}

A field-emission scanning electron microscope (FE-SEM), part of a CrossBeam XB 1540 (Carl Zeiss, Oberkochen, Germany), with a resolution of $1.1 \mathrm{~nm}$, was used for ultra-structural feature characterizations of the $3 \mathrm{D}$ tissue culture. To avoid charging, the sample was coated with a thin layer of chromium (a few nm), which does not alter the morphology of the tissue. An electron beam energy of $20 \mathrm{keV}$, which enhances the morphological contrast obtained with secondary electrons, and an aperture of $30 \mu \mathrm{m}$, which generates a narrow beam current for optimum resolution at high magnification, were used for imaging with a positive biased Everhart-Thornley detector.

\section{Data analysis}

All experiments were performed at least in triplicates (biological replicates). Results are expressed as the mean \pm SEM (standard error of mean). Statistical significance between groups was assessed by two-tailed Student's t-test.

\section{Results}

\subsection{Morphological and ultrastructural characterization of the tissue under static and dynamic conditions}

Confocal imaging of stained cryo-sectioned tissues showed a more pronounced expression of villin, a microfilament-associated, actin-binding protein typical of brush-border microvilli (Bretscher and Weber, 1980), when intestinal tissues were cul- tured under dynamic conditions within $\mathrm{MIVO}^{\circledR}$, compared to static cultured tissues (Fig. 3).

Scanning electron microscopy (SEM) generated micrographs of intestinal tissue cultured in the $\mathrm{MIVO}^{\circledR}$ chamber under dynamic conditions (Panels A, B) or under static conditions (Panel C) also showed a well-developed brush-border in tissues grown under dynamic conditions, confirming the confocal villin staining pattern. The high magnification (Fig. 4B) confirms the good condition of the villi structure grown under dynamic conditions.

\subsection{Sugar permeability tests in healthy, dynamic conditions}

The passage of lactulose and mannitol through the EpiIntestinal ${ }^{\mathrm{TM}}$ tissue model cultured under dynamic conditions was analyzed. The percentage of absorbed mannitol was much greater than that of lactulose at each time point (Figure 5) as expected. Moreover, a plateau was reached in both cases; lactulose reached steady state after 90 minutes, while mannitol reached steady state after 210 minutes. Figure S3 ${ }^{1}$ shows lactulose crystals deposited on the surface of the villi after the absorption test.

As shown in Figures 5, S4 and S5 ${ }^{1}$, the kinetics of lactulose absorption were similar at different concentrations, finally reaching a comparable amount of absorbed sugar (i.e., $49.61 \pm 3.95 \mathrm{mg}$ / $\mathrm{mL}$ for the highest concentration and $47.75 \pm 2.09 \mathrm{mg} / \mathrm{mL}$ for the lowest one), while the final values of mannitol absorbed were significantly different for the two concentrations, with a higher plateau value (about double) for the higher concentration. 
A

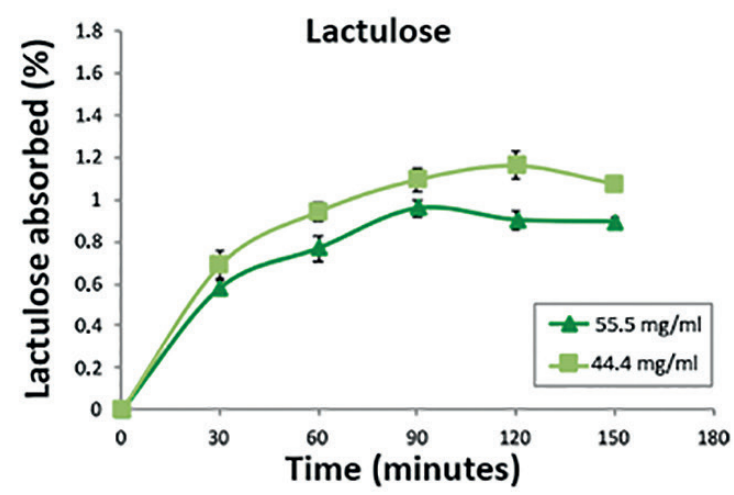

B

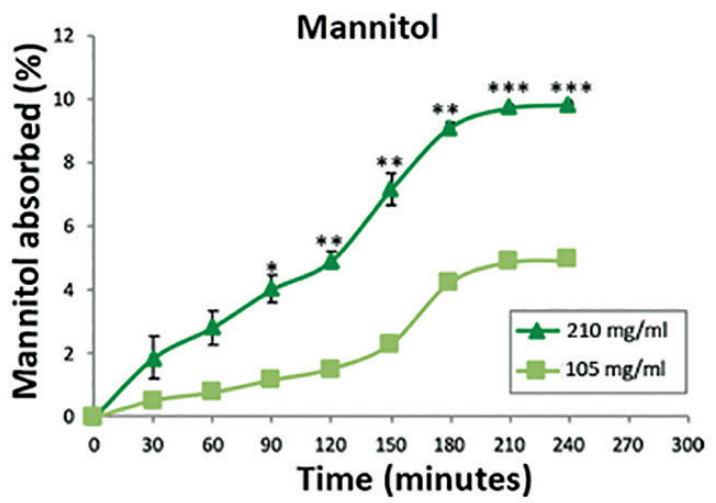

Fig. 5: Comparison of the absorption of two different concentrations of lactulose (A) and mannitol (B) by healthy intestinal tissue in dynamic conditions (MIVO ${ }^{\circledR}$ device)

Values are presented as mean \pm SEM. $N=6$. Student's $t$-test. ${ }^{\star} P<0.05 ;{ }^{* \star} P<0.01 ;{ }^{* \star *} P<0.001$.

\subsection{Sugar permeability tests in pathological conditions}

The sugar permeability tests were repeated after EGTA treatment to evaluate the effects of the progressive disassembly of tight junctions on the two absorption routes. No significant differences in mannitol absorption were observed between EGTA treated and control tissue (Fig. 6A), however, the amount of lactulose passed through the membrane was significantly higher after the EGTA treatment $(\mathrm{p}<0.05)$ (Fig. 6B). Moreover, the LMR measure of the damaged tissues was higher (0.02) than that measured for healthy tissues, in line with clinical results that reported that values increase during the pathogenesis of many gastrointestinal diseases.

The TEER measurement revealed that barrier integrity of all the tissues was within specification with TEER values of $\sim 100$ $\mathrm{Ohm}^{*} \mathrm{~cm}^{2}$ before EGTA treatment. The addition of EGTA to medium for 20 or 45 minutes resulted in a rapid decrease of barrier integrity, as demonstrated by the decrease of TEER by more than $50 \%$ compared to untreated tissues (Fig. 7).

\subsection{Sugar permeability tests after tissue recovery}

To evaluate the intestinal tissues' ability to recover from EGTA treatment, tissue samples were incubated for 24 hours in complete SMI-100 maintenance medium after EGTA treatment. Since EGTA treatment showed no significant difference for mannitol permeation/absorption, only lactulose permeability was assessed in tissues after recovery from EGTA treatment under either static or dynamic conditions.

Interestingly, the pattern of lactulose passage was completely restored only in dynamic conditions (Fig. 6B). When the experiment was carried out in static conditions (Fig. 6C), the EpiIntestinal $^{\mathrm{TM}}$ tissue failed to restore the normal barrier function, maintaining a higher percentage of lactulose passage compared to the healthy tissue before the EGTA treatment.

\section{Discussion}

Intestinal epithelium has a well-organized cellular structure composed of multiple cellular phenotypes. Absorptive enterocytes form a seamless barrier connected by tight junctions that interact with adjacent cells and with intracellular proteins connected to the cytoskeleton (Groschwitz and Hogan, 2009).

Formation of a physiological barrier in most of the in vitro models of intestinal epithelia is detectable by trans-epithelial electrical resistance (TEER) measurements (Srinivasan et al., 2015). In general, this technique provides information about the state of barrier integrity of the intestinal tissue, which directly affects the paracellular passage of molecules, especially large ones like lactulose. However, it is difficult to monitor the passage of small molecules, like mannitol, which also can exploit other mechanisms (e.g. transcellular).

Here we describe an in vitro approach that allows measurement of both paracellular and transcellular intestinal absorption by adapting sugar permeability tests commonly used in the clinic. Modeling pathological conditions by means of calcium chelation allows comparison of the sugar translocation capacity between healthy, diseased, and recovered tissue from the apical to the basolateral surface.

We show that the overall percentage of mannitol passed through the intestinal tissue is higher than that of lactulose, which is in agreement with historical and clinical data (Bijlsma et al., 1995; Ukabam and Cooper, 1984). This data is indicative of the relevance of the model for permeation studies. While paracellular absorption is strictly related to the integrity of the cellular tight junctions, transcellular absorption depends on the available cellular surface area, which is quite large in the model due to the presence of folds and villi-like structures.

It is noteworthy that the percentage of lactulose permeation in two hours reached the same plateau value starting from different 


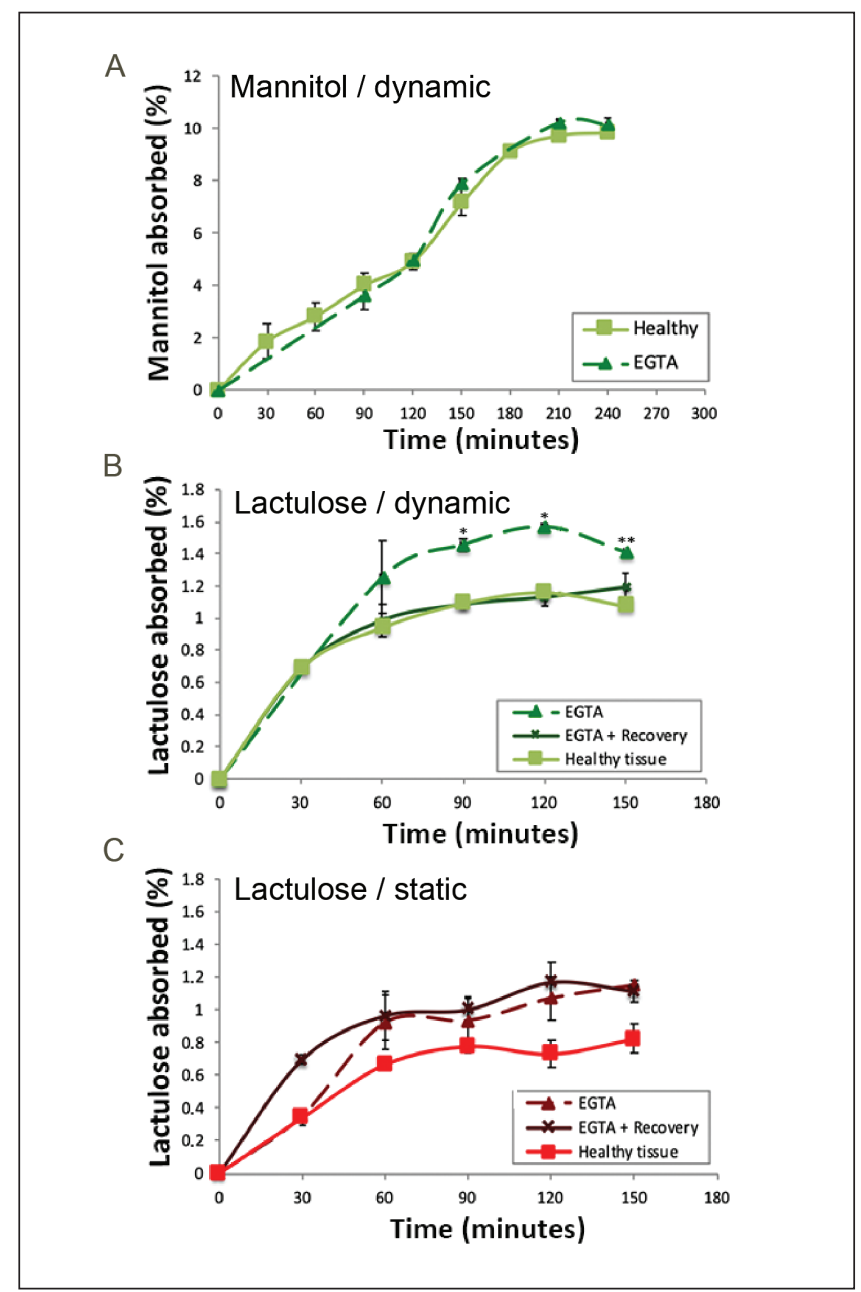

Fig. 6: Lactulose but not mannitol absorption increases in the absence of $\mathrm{Ca}^{2+}$ and is recovered after supplementation of $\mathrm{Ca}^{2+}$ only in dynamic culture conditions

(A) Absorption of mannitol in the absence and presence of EGTA in dynamic culture conditions (MIVO ${ }^{\circledR}$ chamber); (B) Absorption of lactulose in the absence and presence of EGTA and after recovery in EGTA-free medium in dynamic culture conditions (MIVO ${ }^{\circledR}$ chamber); (C) Absorption of lactulose in the absence and presence of EGTA and after recovery in EGTA-free medium in static culture conditions. $210 \mathrm{mg} / \mathrm{mL}$ mannitol; $44.4 \mathrm{mg} / \mathrm{mL}$ lactulose. Values are presented as mean \pm SEM. $N=6$. Student's $t$-test. ${ }^{*} P<0.05 ;{ }^{* *} \mathrm{P}<0.01$.

concentrations in terms of the absorbed absolute lactulose value. However, the levels of mannitol passed though the tissue were significantly different and proportional to the starting concentrations in the donor chamber. A possible explanation could be that the human intestinal tissue adopts a controlled passive diffusion process aimed at keeping the amount of lactulose on the blood side constant. In fact, the paracellular passage is mediated by the tight junctions, which are able to dynamically regulate the passage of solutes and molecules. They are programmed to rapidly

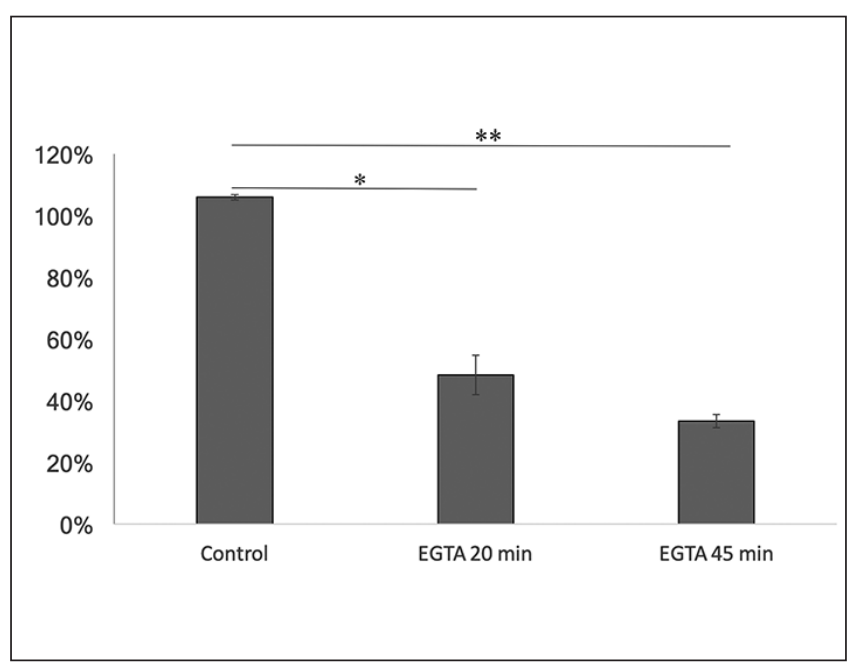

Fig. 7: TEER measurement before and after EGTA treatment Values are presented as mean $\pm S E M . N=2$. Student's $t$-test. ${ }^{\star} P<0.05$; ${ }^{\star \star} P<0.01$.

open and seal the barrier in the event of injury, healing, and other signals (Shen, 2012; Chelakkot et al., 2018). Mannitol absorption, on the other hand, was much faster for the higher sugar concentration, in accordance with the Michaelis-Menten kinetic of a typical enzyme-catalyzed reaction as reported previously (Diallinas and De Koning, 2014; Vivian and Polli, 2014).

Although lactulose and mannitol mainly pass through different intestinal routes, several studies have shown that a small molecule like mannitol can be transported also through the intercellular spaces (Kotzé et al., 1998). This occurs because the tight junctions in the intestinal epithelium that regulate paracellular transport are size-selective (Maiti, 2017). In fact, a non-metabolized probe penetrates the intestinal barrier with a rate proportional to its molecular size (Hollander, 1993).

Therefore, the routes available for absorption of large molecules (e.g. lactulose) are obviously fewer than those available for smaller molecules (e.g. mannitol). Accordingly, it is reported that a higher amount of mannitol is generally absorbed $(15 \%)$ compared to lactulose $(0.1 \%)$ in normal patients (Ukabam and Cooper, 1984). In fact, there are historical data that indicate that mannitol can be absorbed via both intercellular spaces (Hollander, 1992; Menard et al., 2012) and transcellular permeation mechanisms (Menzies, 1984; Musa et al., 2019; Paroni et al., 2006) involving numerous small water pores situated in the cell membranes of the mucosal enterocytes. However, to our knowledge, there are no studies, prior to our work, on the in vitro intestinal mechanisms of passage of mannitol and lactulose using 3D primary cell-based small intestinal tissues (commercially available and highly reproducible) in a fluid-dynamic environment resembling the physiological microenvironment of human intestinal tissues.

Our results show that the absorption routes of the two sugars are different, in line with the clinical knowledge that routinely uses the LMR index calculated from urinary recovery to identify 
possible intestinal damage of patients. In particular, we demonstrate the utility of an in vitro intestinal tissue model using lactulose as a marker of the paracellular pathway and an indicator of intestinal barrier integrity and mannitol as marker of the transcellular pathway indicative of mucosal absorption. At the end of the permeation test, the LMR value was derived from the ratio of the plateau values of lactulose (L) and mannitol (M), as reported in the literature (Dastych et al., 2008). The measured LMR was 0.01 , which is comparable with values of healthy intestinal tissue reported in clinical practice (Barboza Junior et al., 1999; Dastych et al., 2008). These novel outcomes, therefore, provide the theoretical basis for using the LMR index as an indirect measure of the permeability of intestinal tissue at steady state in healthy intestinal tissue.

A compromised epithelial barrier was generated using EGTA to amplify the differences between the paracellular and transcellular routes. After EGTA treatment, the amount of lactulose passing through the membrane increased significantly. It must be noted that the rate of permeation of molecules is also influenced by the leakiness of the tight junctions (i.e., paracellular path) caused by EGTA treatment. The alteration of these junctions, which increases the permeability of the epithelial barrier, occurs through the phosphorylation of myosin and the contraction of the actin-myosin complexes, with a progressive disassembly of tight junctions and related epithelial barrier integrity (Rothen-Rutishauser et al., 2002; Tsukita et al., 2001).

On the contrary, no significant differences were observed for mannitol absorption, due to the apparent minor importance of the paracellular route in the absorption of mannitol, which is indeed mainly determined by the high cell surface area of the EpiIntestinal $^{\text {TM }}$ tissue that remains constant in healthy and damaged tissues (Shaikh et al., 2015). In intestinal absorption assays based on CaCo-2 cells, which are known to have a much stronger barrier property than the small intestine, mannitol is frequently used as a low permeability marker molecule to monitor barrier integrity. When the intestinal barrier is compromised due to tight junction modulation/disruption as a result of drug toxicity or pathological conditions such as simulated by calcium chelation, mannitol permeability is expected to increase.

The intestinal epithelium is known to be a highly specialized tissue, which consists of different cell phenotypes, including a stem cell population that contributes to a continuous turnover of the villi-like structure. Several studies have shown the ability of the intestinal epithelium to rapidly re-establish its integrity following injury (Lacy, 1988). In our hands, after calcium depletion treatment, the pattern of lactulose passage was comparable with that of healthy tissue. However, tissue recovery was completely restored only in dynamic conditions within the $\mathrm{MIVO}^{\circledR}$ chamber. This is probably due to the beneficial role of the dynamic fluid flow beneath the membrane, which helps to remove the accumulation of waste while providing a more physiological niche and supporting a healthy tissue status. This in turn improves molecular transport and cellular turnover, which significantly contributes to the healing of the tissue (Kim et al., 2016). In fact, the accumulation of waste products, mainly lactate, which occurs in 2-dimensional (2D) static cultures, can lead to an acidification of the $\mathrm{pH}$ in the tissue microenvironment, which can impair cell growth (Chen et al., 2010).

Altered barrier permeability is the basis of pathogenesis of many gastroenterological and non-gastrointestinal pathologies such as infectious enterocolitis, chronic intestinal inflammatory diseases (IBD), irritable bowel syndrome (IBS), bacterial overgrowth syndrome of the small intestine (SIBO), celiac disease, and even atopic manifestations (Scaldaferri et al., 2012). Thus, in vitro modeling of a fluid-dynamic human intestine environment with disease-like perturbations offers an innovative and useful approach to the in vitro testing of potential therapeutic drugs on the recovery of the physiological function of the intestinal barrier (Tab. S11).

In conclusion, the results reported in this study indicate that the $\mathrm{MIVO}^{\circledR}$ fluidic flow system combined with the EpiIntestinal $^{\mathrm{TM}}$ platform can be a valuable in vitro model of the healthy and pathological small intestinal barrier to study the passage of molecules by discriminating the two main intestinal absorption mechanisms. Moreover, this system could become a useful tool to test the effects of therapies and drugs on intestinal tissue barrier function.

\section{References}

Ayehunie, S., Landry, T., Stevens, Z. et al. (2018). Human primary cell-based organotypic microtissues for modeling small intestinal drug absorption. Pharm Res 35, 72. doi:10.1007/ s11095-018-2362-0

Barboza Junior, M. S., Silva, T. M., Guerrant, R. L. et al. (1999). Measurement of intestinal permeability using mannitol and lactulose in children with diarrheal diseases. Braz J Med Biol Res 32, 1499-1504. doi:10.1590/S0100-879X1999001200008

Benam, K. H., Dauth, S., Hassell, B. et al. (2015). Engineered in vitro disease models. Annu Rev Pathol 10, 195-262. doi:10.1146/annurev-pathol-012414-040418

Bijlsma, P. B., Peeters, R. A., Groot, J. A. et al. (1995). Differential in vivo and in vitro intestinal permeability to lactulose and mannitol in animals and humans: A hypothesis. Gastroenterology 108, 687-696. doi:10.1016/0016-5085(95)90440-9

Bjarnason, I., MacPherson, A. and Hollander, D. (1995). Intestinal permeability: An overview. Gastroenterology 108, 15661581. doi:10.1016/0016-5085(95)90708-4

Bretscher, A. and Weber, K. (1980). Villin is a major protein of the microvillus cytoskeleton which binds both $G$ and $\mathrm{F}$ actin in a calcium-dependent manner. Cell 20, 839-847. doi:10.1016/0092-8674(80)90330-X

Cao, X., Gibbs, S. T., Fang, L. et al. (2006). Why is it challenging to predict intestinal drug absorption and oral bioavailability in human using rat model. Pharm Res 23, 1675-1686. doi:10.1007/s11095-006-9041-2

Chelakkot, C., Ghim, J. and Ryu, S. H. (2018). Mechanisms regulating intestinal barrier integrity and its pathological implications. Exp Mol Med 50, 103. doi:10.1038/s12276-018-0126-X

Chen, X., Chen, A., Woo, T. L. et al. (2010). Investigations in- 
to the metabolism of two-dimensional colony and suspended microcarrier cultures of human embryonic stem cells in serum-free media. Stem Cells Dev 19, 1781-1792. doi:10.1089/ scd.2010.0077

Collares-Buzato, C. B., McEwan, G. T., Jepson, M. A. et al. (1994). Paracellular barrier and junctional protein distribution depend on basolateral extracellular $\mathrm{Ca}^{2+}$ in cultured epithelia. Biochim Biophys Acta 1222, 147-158. doi:10.1016/01674889(94)90163-5

Dastych, M., Dastych, M., Novotná, H. et al. (2008). Lactulose/mannitol test and specificity, sensitivity, and area under curve of intestinal permeability parameters in patients with liver cirrhosis and Crohn's disease. Dig Dis Sci 53, 2789-2792. doi:10.1007/s10620-007-0184-8

Diallinas, G. and De Koning, H. P. (2014). Understanding transporter specificity. Front Pharmacol 5, 207. doi:10.3389/fphar. 2014.00207

Ganapathy, V. (2012). Chapter 59 - Protein digestion and absorption. In L. R. Johnson, F. K. Ghishan, J. D. Kaunitz et al. (eds.), Physiology of the Gastrointestinal Tract (1595-1623). $5^{\text {th }}$ edition. Academic Press. doi:10.1016/B978-0-12-3820266.00059-2

Groschwitz, K. R. and Hogan, S. P. (2009). Intestinal barrier function: Molecular regulation and disease pathogenesis. $\mathrm{J} \mathrm{Al}$ lergy Clin Immunol 124, 3-20. doi:10.1016/j.jaci.2009.05.038

Gupta, V., Doshi, N. and Mitragotri, S. (2013). Permeation of insulin, calcitonin and exenatide across caco-2 monolayers: Measurement using a rapid, 3-day system. PLoS One 8, e57136. doi:10.1371/journal.pone.0057136

Halme, L., Turunen, U. and Tuominen, J. (2000). Comparison of iohexol and lactulose-mannitol tests as markers of disease activity in patients with inflammatory bowel disease. Scand J Clin Lab Invest 60, 695-701. doi:10.1080/00365510050216420

Hollander, D. (1992). The intestinal permeability barrier: A hypothesis as to its regulation and involvement in Crohn's disease. Scand J Gastroenterol 27, 721-726. doi:10. 3109/00365529209011172

Holmes, A. M., Creton, S. and Chapman, K. (2010). Working in partnership to advance the $3 \mathrm{Rs}$ in toxicity testing. Toxicology 267, 14-19. doi:10.1016/j.tox.2009.11.006

Huang, Y. and Adams, M. (2003). An in vitro model for investigating intestinal adhesion of potential dairy propionibacteria probiotic strains using cell line C2BBe1. Lett Appl Microbiol 36, 213-216. doi:10.1046/j.1472-765X.2003.01303.x

Kim, H. J., Lee, J., Choi, J.-H. et al. (2016). Co-culture of living microbiome with microengineered human intestinal villi in a gut-on-a-chip microfluidic device. J Vis Exp 114, e54344. doi: $10.3791 / 54344$

Kotzé, A. F., Lueßen, H. L., de Leeuw, B. J. et al. (1998). Comparison of the effect of different chitosan salts and n-trimethyl chitosan chloride on the permeability of intestinal epithelial cells (Caco-2). J Control Release 51, 35-46. doi:10.1016/ S0168-3659(97)00154-5

Lacy, E. R. (1988). Epithelial restitution in the gastrointestinal tract. J Clin Gastroenterol 10, Suppl 1, S72-S77. doi:10.

\section{7/00004836-198812001-00012}

Le Ferrec, E., Chesne, C., Artusson, P. et al. (2001). In vitro models of the intestinal barrier: The report and recommendations of ECVAM workshop 46. Altern Lab Anim 29, 649-668. doi:10.1177/026119290102900604

Maiti, S. (2017). Chapter 6 - Nanometric biopolymer devices for oral delivery of macromolecules with clinical significance. In A. M. Grumezescu (ed.), Multifunctional Systems for Combined Delivery, Biosensing and Diagnostics (109-138). Elsevier. doi:10.1016/B978-0-323-52725-5.00006-X

Markus J., Landry T., Klausner K. et al. (2017). Lab to lab reproa ducibility in the production of physiologically relevant reconstructed small intestine tissue for in vitro testing of toxicity, permeation and inflammation. Toxicol Lett 280, Suppl 1, S324. doi:10.1016/j.toxlet.2017.08.077

Marrella, A., Iafisco, M., Adamiano, A. et al. (2018). A combined low-frequency electromagnetic and fluidic stimulation for a controlled drug release from superparamagnetic calcium phosphate nanoparticles: Potential application for cardiovascular diseases. J R Soc Interface 15, 20180236. doi:10.1098/ rsif.2018.0236

Menard, S., Lebreton, C., Schumann, M. et al. (2012). Paracelf lular versus transcellular intestinal permeability to gliadin peptides in active celiac disease. Am J Pathol 180, 608-615. doi:10.1016/j.ajpath.2011.10.019

Menzies, I. (1984). Transmucosal passage of inert molecules in health and disease. In Skahauge, E. and Heintz, K. (eds), Intestinal Absorption and Excretion. Falk Symposium 36, 527-543. http://www.cmcwtrl.in/publications/5-1983-FalkSymposium-36.pdf

Musa, M. A., Kabir, M., Hossain, M. I. et al. (2019). Measurement of intestinal permeability using lactulose and mannitol with conventional five hours and shortened two hours urine collection by two different methods: HPAE-PAD and LC-MSMS. PLoS One 14, e0220397. doi:10.1371/journal. pone. 0220397

Paroni, R., Fermo, I., Molteni, L. et al. (2006). Lactulose and minnitol intestinal permeability by capillary electrophoresis. J Chromatography $B$ 834, 183-187. doi:10.1016/j. jchromb.2006.02.050

Rothen-Rutishauser, B., Riesen, F. K., Braun, A. et al. (2002). Dynamics of tight and adherens junctions under EGTA treatment. J Membr Biol 188, 151-162. doi:10.1007/s00232-0010182-2

Russell, W. M. S., Burch, R. L. and Hume, C. W. (1959). The Principles of Humane Experimental Technique. London, UK: Methuen.

Scaldaferri, F., Pizzoferrato, M., Gerardi, V. et al. (2012). The gut barrier: New acquisitions and therapeutic approaches. J Clin Gastroenterol 46, Suppl, S12-S17. doi:10.1097/MCG.0b013 e31826ae849

Sequeira, I. R., Lentle, R. G., Kruger, M. C. et al. (2014). Standardising the lactulose mannitol test of gut permeability to minimise error and promote comparability. PLoS One 9, e99256. doi:10.1371/journal.pone.0099256 
Shaikh, M., Rajan, K., Forsyth, C. B. et al. (2015). Simultaneous gas-chromatographic urinary measurement of sugar probes to assess intestinal permeability: Use of time course analysis to optimize its use to assess regional gut permeability. Clin Chim Acta 442, 24-32. doi:10.1016/j.cca.2014.12.040

Shen, L. (2012). Tight junctions on the move: Molecular mechanisms for epithelial barrier regulation. Ann N Y Acad Sci 1258, 9-18. doi:10.1111/j.1749-6632.2012.06613.x

Söderholm, J. D., Olaison, G., Lindberg, E. et al. (1999). Different intestinal permeability patterns in relatives and spouses of patients with Crohn's disease: An inherited defect in mucosal defence? Gut 44, 96-100. doi:10.1136/gut.44.1.96

Srinivasan, B., Kolli, A. R., Esch, M. B. et al. (2015). TEER measurement techniques for in vitro barrier model systems. $J$ Lab Autom 20, 107-126. doi:10.1177/2211068214561025

Stewart, B. H., Chan, O. H., Lu, R. H. et al. (1995). Comparison of intestinal permeabilities determined in multiple in vitro and in situ models: Relationship to absorption in humans. Pharm Res 12, 693-699. doi:10.1023/A:1016207525186

Tavelin, S., Taipalensuu, J., Söderberg, L. et al. (2003). Predic- tion of the oral absorption of low-permeability drugs using small intestine-like 2/4/A1 cell monolayers. Pharm Res 20, 397-405. doi:10.1023/A:1022699920043

Tsukita, S., Furuse, M. and Itoh, M. (2001). Multifunctional strands in tight junctions. Nat Rev Mol Cell Biol 2, 285-293. doi: $10.1038 / 35067088$

Ukabam, S. O. and Cooper, B. T. (1984). Small intestinal permeability to mannitol, lactulose, and polyethylene glycol 400 in celiac disease. Dig Dis Sci 29, 809-816. doi:10.1007/ BF01318423

Vivian, D. and Polli, J. E. (2014). Mechanistic interpretation of conventional michaelis-menten parameters in a transporter system. Eur J Pharm Sci 64, 44-52. doi:10.1016/j. ejps.2014.08.007

\section{Conflict of interest}

SS and MA are cofounders and shareholders of React4life S.r.l. JM is an employee of MatTek in Vitro Life Science Laboratories; TL and SA are employees of MatTek Corporation. 